\title{
Cerebrospinal fluid kappa free light chains as biomarker in multiple sclerosis - from diagnosis to prediction of disease activity
}

\author{
Harald Hegen (iD · Klaus Berek · Florian Deisenhammer
}

Received: 14 September 2021 / Accepted: 3 January 2022 / Published online: 8 February 2022

(C) The Author(s) 2022

\begin{abstract}
Summary Multiple sclerosis (MS) is a chronic immune-mediated disorder of the central nervous system that shows a high interindividual heterogeneity, which frequently poses challenges regarding diagnosis and prediction of disease activity. In this context, evidence of intrathecal inflammation provides an important information and might be captured by kappa free light chains ( $\kappa-F L C)$ in the cerebrospinal fluid (CSF). In this review, we provide an overview on what is currently known about $\kappa$-FLC, its historical development, the available assays and current evidence on its diagnostic and prognostic value in MS. Briefly, intrathecal $\kappa$-FLC synthesis reaches similar diagnostic accuracy compared to the well-established CSF-restricted oligoclonal bands (OCB) to identify patients with MS, and recent studies even depict its value for prediction of early MS disease activity. Furthermore, detection of $\kappa$-FLC has significant methodological advantages in comparison to OCB detection.
\end{abstract}

Keywords Clinically isolated syndrome - FLC index . Oligoclonal bands · Progression · Relapse

\section{Introduction}

Multiple sclerosis (MS) is a chronic inflammatory immune-mediated disease of the central nervous system (CNS) that mainly affects young adults and bears the risk of physical and cognitive disability [1].

Diagnosis of MS requires the combination of clinical signs and symptoms with paraclinical findings obtained by magnetic resonance imaging (MRI) and cerebrospinal fluid (CSF) analysis [2]. Evidence of

H. Hegen, PD, MD, PhD $(\bowtie) \cdot K$. Berek · F. Deisenhammer Department of Neurology, Medical University of Innsbruck, Anichstr. 35, 6020 Innsbruck, Austria

harald.hegen@i-med.ac.at intrathecal immunoglobulin G (IgG) synthesis in the CSF, although not specific for MS, increases diagnostic certainty in the appropriate clinical setting [3] and substitutes for dissemination in time according to current diagnostic criteria [2].

Besides establishing MS diagnosis, one of the main challenges for neurologists counselling patients with MS is weighing benefits versus risks of certain diseasemodifying therapies (DMTs) [4]. An ever-increasing number of DMTs have been proven to reduce the number of relapses, accumulation of disability and brain MRI activity [5] and current treatment concepts recognize the importance of early treatment towards suppressing disease activity below the level of detectability [6]. However, the interindividual courses of MS are extremely variable [7] and there is also a certain risk for treatment-associated adverse events. Since criteria guiding decisions when to start treatment in early MS and, in case, whether to choose a moderately or a highly efficacious DMT are still controversially debated, there is an urgent need of biomarkers to predict disease activity $[4,8]$. So far, the number of brain MRI lesions and the presence of intrathecal IgG synthesis in the CSF imply some prognostic value [9].

As depicted above, the value of CSF analysis for diagnosis of MS and for prediction of disease activity after the first demyelinating CNS event is unquestioned. However, for the detection of intrathecal IgG synthesis as a marker for intrathecal B cell activity, several different laboratory methods have been developed in the last half century. Quantitative methods that require the measurement of IgG concentrations in CSF and serum followed by calculation of certain formulae such as IgG index [10], Reiber [11] or Auer \& Hegen formulae [12] referring patient's individual values to a predefined upper normal limit are mainly hampered by their low sensitivity. The detection of oligoclonal 
IgG bands (OCB) by isoelectric focusing (IEF) followed by immunoblotting is nowadays the gold standard. This technique compares paired CSF and blood samples of each individual patient. Intrathecal IgG synthesis is present if OCB are present in CSF without corresponding bands in serum [13]. It ensures a high diagnostic sensitivity and specificity both of approximately $90 \%$ [14]. However, this method enables only a qualitative determination of intrathecal IgG synthesis (i.e., returns either a positive or a negative result), is technically demanding, time-consuming, costly and rater-dependent [13].

\section{Kappa free light chains in the CSF as an emerging biomarker}

Besides intact immunoglobulins that consist of light chains and heavy chains bound together via disulfide bonds and noncovalent interactions [15], B cells also produce light chains in $10-40 \%$ excess over heavy chains and secrete them as free forms into the blood circulation [16]. These free light chains (FLC) have a molecular weight of approximately $24 \mathrm{kD}$ and consists of two immunoglobulin domains, a constant region that specifies the isotype of free light chain (either $\kappa$ or $\lambda$ ) and a variable domain (Fig. 1; [15]). If bound, the variable light chain domain is part of the immunoglobulin antigen binding site; the function in the free forms is not fully elucidated [16]. $\kappa$-FLC exist mainly in the form of monomers, whereas $\lambda$-FLC are present as covalent dimers [16]. In the last few years, a multitude of studies have highlighted the value of $\kappa$-FLC in CSF as another biomarker-instead of immunoglobulins-for intrathecal B cell activity in patients with MS, not least due to significant methodological advantages.

\section{History of FLC detection}

FLCs were discovered more than 150 years ago, when in 1847 Henry Bence Jones described a protein in the urine of a patient with severe bone pain and fractures that precipitated upon addition of nitric acid [17]. The so-called Bence Jones proteins evolved to an important diagnostic marker for patients with multiple myeloma. More than 100 years after its discovery, the Bence Jones protein was identified as monoclonal FLC [18]. Developments in laboratory methods brought up protein electrophoresis and immunofixation electrophoresis; however, these methods still had limited sensitivity so that low level FLC under physiological or oligosecretory conditions, e.g., in immune-mediated diseases, were not detectable, and allowed only qualitative determination [18]. Attempts to quantify FLC were initially hindered by difficulties of producing antibodies specific to FLC that do not cross-react with light chains bound in intact immunoglobulins. The breakthrough was achieved in 2001 by Bradwell and coworkers who dissociated light chains from heavy chains and then raised antibodies directed against unique epitopes on FLC that are normally "hidden" in the conformational structure of an intact immunoglobulin [19]. These anti-human FLC-specific antibodies could then be used to develop assays that exclusively detect FLC at least a hundred times more sensitive than previous methods with detection limits down to approximately $1 \mathrm{mg} / \mathrm{L}$. Nowadays, FLC can be measured in serum as well as in CSF by use of two types of detection antibodies: either polyclonal [19] or monoclonal [20] detection antibodies.

\section{Elevated $\kappa-F L C$ in the CSF of patients with MS}

$\kappa$-FLC in the CSF-similar to immunoglobulins or other proteins-originate either from blood by diffusion across the blood-CSF barrier or are produced within the intrathecal compartment under pathological conditions [21]. Conceptually, it seems necessary to determine the locally synthesized $\kappa$-FLC fraction separate from the blood-derived fraction (as it is also done for IgG). Most studies calculated the $\kappa$-FLC index that considers the CSF/serum albumin quotient $\left(\mathrm{Q}_{\text {alb }}\right)$ which is an established marker for the blood-CSF barrier function [22] and corrects for the absolute serum $\kappa$-FLC level. The $\kappa$-FLC index is determined by following formula $[23,24]$ :

$$
\kappa-\text { FLC index }=\frac{\kappa-\mathrm{FLC}_{\mathrm{CSF}} / \kappa-\mathrm{FLC}_{\mathrm{Serum}}}{Q_{\mathrm{alb}}}
$$

It has been consistently shown that the $\kappa$-FLC index reaches a high diagnostic accuracy to identify patients with MS. An overview of current evidence-retrieved in a systematic literature search [23-40]-is provided in Table 1. For the $\kappa$-FLC index, diagnostic sensitivity ranges from 52 to $98 \%$ (weighted average: $87 \%$ ) and specificity ranges from 68 to $100 \%$ (weighted average: $89 \%$ ). For OCB, sensitivity ranges from 37 to $100 \%$ (weighted average: $84 \%$ ) and specificity from 74 to $100 \%$ (weighted average: 90\%). The reported sensitivity of OCB is in accordance with a previous meta-analysis [41]. Applying a difference-in-differences model showed that the mean difference of diagnostic sensitivity between $\kappa$-FLC index and OCB was $+2 \%$ and of specificity was $-2 \%$, i.e., overall the diagnostic accuracy of $\kappa$-FLC index and OCB was equal.

The wide range of diagnostic sensitivity and specificity for both the $\kappa$-FLC index and OCB arises from a certain heterogeneity between studies. It is evident that specificity of $\kappa$-FLC index is lowered when patients with inflammatory neurological disease (IND) were included into the control group. $\mathrm{k}$-FLC in CSF are-similar to CSF-restricted OCB-a sign of intrathecal inflammation and thus can support the diagnosis of MS, but they are not specific for MS. The spectrum of diseases which show intrathecal $\kappa$-FLC synthesis is probably identical to that with CSF-restricted OCB, even though studies on the frequency of intrathecal $\kappa$-FLC synthesis in other neurological 


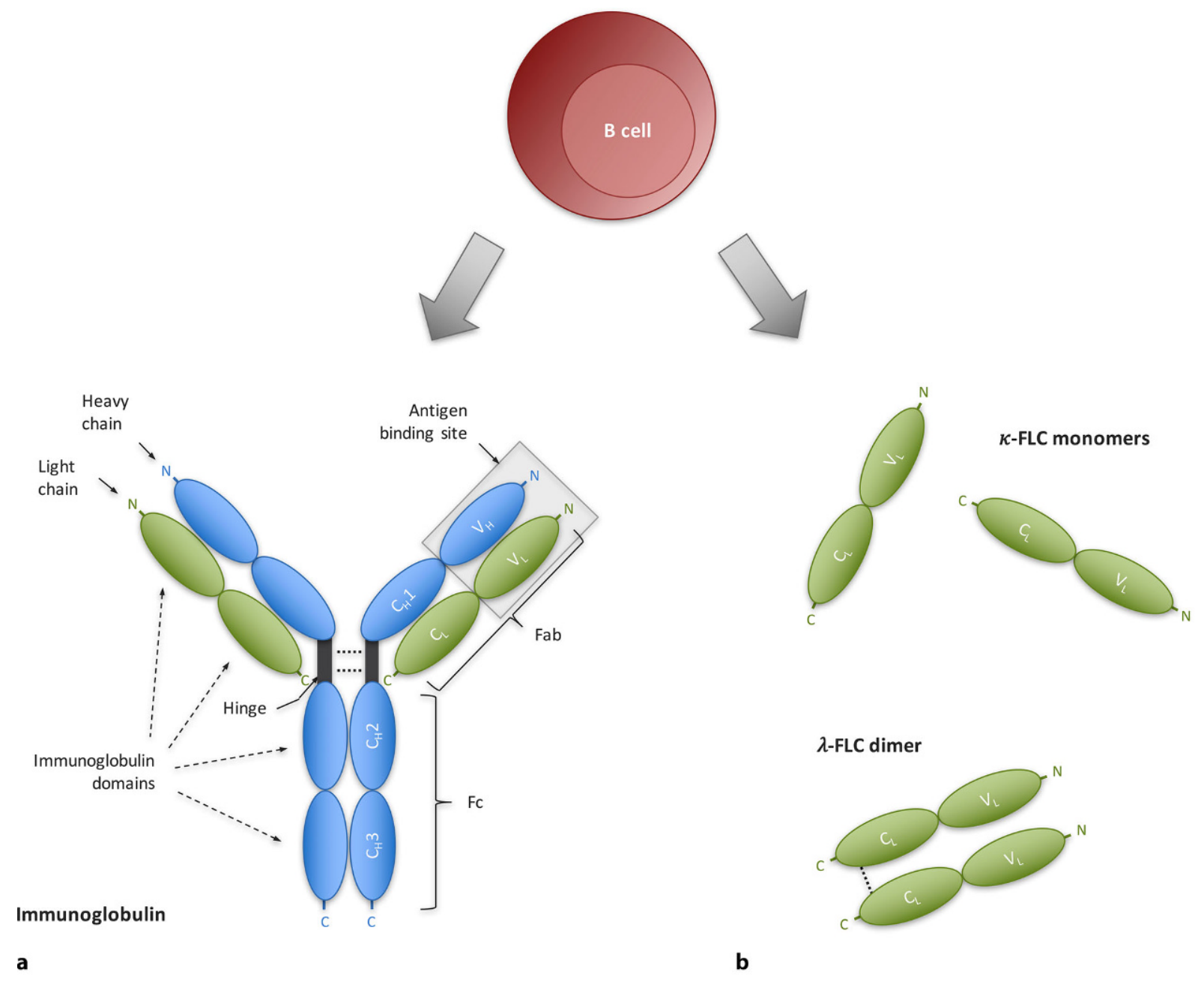

Detection methods:

- Quantitative: Measurement of immunoglobulin concentrations in CSF and serum, e.g., by turbidimetry or nephelometry, and calculation of the intrathecal fraction by, e.g., Reiber, Auer \& Hegen formulae or IgG index

- Qualitative: Isoelectric focusing followed by immunofixation revealing oligoclonal IgG bands

Fig. 1 Schematic illustration of the molecular structure of immunoglobulins and free light chains. B cells produce (a) intact immunoglobulins and (b) in excess free light chains. Both immunoglobulins and FLC serve as biomarker for B cell activity. a Immunoglobulins consist of two identical heavy chains (b/ue) and two identical light chains (green). Each heavy chain consists of four immunoglobulin domains linked by a hinge region. Differences in the structure of the constant regions $\left(\mathrm{C}_{\mathrm{H}} 1, \mathrm{C}_{\mathrm{H}} 2\right.$ and $\left.\mathrm{C}_{\mathrm{H}} 3\right)$ determine the isotype $(\mathrm{IgG}, \mathrm{M}, \mathrm{A}, \mathrm{D}, \mathrm{E})$ and subclass of the immunoglobulin (e.g., IgG1-4), while the variable domain $\left(\mathrm{V}_{\mathrm{H}}\right)$ contributes to the antigen binding site. Each light chain consists of two immunoglobulin domains. Differences in the structure of the constant region $\left(C_{L}\right)$ determine the isotype of free light chain (either $\kappa$ or $\lambda$ ), while the variable domain $\left(V_{L}\right)$ contributes to the antigen binding site. Accord-
- Quantitative: Measurement of FLC in CSF and serum, e.g., by turbidimetry or nephelometry, and calculation of the intrathecal fraction by, e.g., FLC index

- Qualitative: Not relevant

ingly, both the heavy chains and light chains form the aminoterminal variable $(\mathrm{V})$ regions responsible for antigen recognition; the carboxyl-terminal constant $(C)$ regions mediate effector functions. b Free light chains show the same structure as light chains bound within the intact immunoglobulin. FLC have a molecular weight of approximately $24 \mathrm{kD}$ and consist of the two immunoglobulin domains $C_{L}$ and $V_{L}$. Differences in the structure of the constant region $\left(\mathrm{C}_{\mathrm{L}}\right)$ determine the isotype of the free light chain (either $\kappa$ or $\lambda$ ). Whereas $\kappa$-FLC mainly exist in the form of monomers, $\lambda$-FLC are present as covalent dimers. $C_{H}$ constant heavy chain domain, $C_{L}$ constant light chain domain, Fab fragment antibody binding, $F C$ fragment crystallisable, $F L C$ free light chain, $V_{H}$ variable heavy chain domain, $V_{L}$ variable light chain domain 
disease are still rare. Apart from a mixture of different IND as part of control populations (Table 1) that had $\kappa$-FLC synthesis in up to $32 \%$, dedicated disease-specific studies exist only for a few entities, e.g., neuroborreliosis [43, 44].

\section{$\kappa-F L C$ index associated with early MS disease activity}

There are only a few studies on the predictive value of the $\kappa$-FLC index in MS. An overview is given in Table 2. These studies reported that the presence of intrathecal $\kappa-F L C$ synthesis is associated with conversion from CIS to MS [45-49] and that the $\kappa$-FLC index predicted the time to conversion to MS as well as disability progression [49, 50]. However, these studies had some methodological limitations. A multivariate approach that considers other already known risk factors especially MRI activity is critical to identify the independent prognostic effect of the $\kappa$-FLC index and to weigh its impact on the outcome.

There is one recent study that fulfills these requirements providing class II evidence that in patients with early MS, high $\kappa$-FLC index is an independent risk factor for early second clinical attack. A cohort of 88 patients with a first CNS demyelinating event (mostly monofocal, $45 \%$ myelitis, $30 \%$ optic neuritis, $24 \%$ affection of brainstem/cerebellum), at a mean age of 33 years and with a female predominance of $68 \%$ were followed over 4 years. In all, 38 (43\%) patients converted to clinically definite MS (CDMS) within the observation period. In multivariate Cox regression analysis adjusting for age, sex, MRI lesion load and activity at baseline, administration of corticosteroids at baseline and DMT during follow-up revealed that $\kappa$-FLC index predicts time to second clinical attack. This study showed that patients with $\kappa$-FLC index $>100$ at baseline had a twice as high probability for a second clinical attack within 12 months than patients with low $\kappa$-FLC index; within 24 months, the chance in patients with high $\kappa$-FLC index was 4 times as high as in patients with low $\kappa$-FLC index. The median time to second attack was 11 months in patients with high $\kappa$-FLC index, whereas 36 months in those with low $\kappa$-FLC index [52].

\section{Advantages of $\kappa-F L C$ index compared to OCB}

Current evidence suggests that determination of $\kappa$-FLC index has some advantages over OCB detection. Even though it seems that there is no relevant difference with regard to diagnostic accuracy (Table 1), $\kappa$-FLC can be easily measured by nephelometry which is-in contrast to the detection of OCB-a reliable, labor-saving and cost-efficient method [20]. Moreover, $\kappa$-FLC index returns a metric result covering a range from approximately 1 up to 500 [23], i.e., it is a quantitative parameter, while OCB status is dichotomous returning either a positive or negative result as assessed by visual inspection [13]. The advantage of a metric result seems important especially for predicting disease activity. In the most recent study on the predictive value of $\kappa$-FLC index-as previously mentioned [52]—which included patients with a first CNS demyelinating event, OCB were detected in $95 \%$ of patients who converted to CDMS during the 4-year follow-up (CDMS converters), whereas nonconverters were OCB positive also in $86 \%$ of cases. As a continuous variable, $\kappa$-FLC index overcame the weak performance of OCB by further stratification. $\kappa$-FLC index also significantly differed between OCBpositive CDMS converters and OCB-positive nonconverters and predicted CDMS conversion also within the cohort of OCB-positive patients [52]. Despite these promising results and clear methodological advantages of $\kappa$-FLC index over OCB, the latter is still considered the gold standard. Before $\kappa$-FLC index might be introduced into clinical routine, a few issues still need to be clarified, e.g., whether calculation of intrathecal $\kappa$-FLC synthesis is superior to determination of absolute $\kappa$-FLC concentrations in CSF or which cut-off should be applied. These two open issues are discussed in the following.

\section{Open issues}

\section{Determining $\kappa$-FLC index or absolute CSF $\kappa$-FLC values}

As mentioned above, one might argue that determining the locally synthesized fraction of $\kappa$-FLC separate from the blood-derived fraction is necessary to capture an intrathecal inflammatory process. And indeed, the majority of studies used the $\kappa$-FLC index (Table 1) or calculated an intrathecal $\kappa$-FLC fraction by empirically determined $\mathrm{Q}_{\mathrm{alb}}$-dependent reference limits $[25,43,51,53]$, whereas some studies included the CSF/serum $\kappa$-FLC ratio ( $\left.\mathrm{Q}_{\kappa-\mathrm{FLC}}\right)$ [30, 38, 54-58]. Other authors determined the absolute CSF $\kappa$-FLC concentrations [31] arguing that the contribution of bloodderived FLC to the total CSF FLC concentration is low in cases with intrathecal synthesis. In fact, the intrathecal fraction of $\kappa$-FLC is greater than $80 \%$ in most MS patients [23], and around 15\% of CIS/MS patients showed even higher absolute $\kappa$-FLC concentrations in CSF than in serum that proves an intrathecal synthesis per se [59]. To further elaborate this research question, a recent study separated patients into low and high CSF $\kappa$-FLC categories (based on median values) and observed that CSF $\kappa$-FLC concentration, $\mathrm{Q}_{\kappa-\mathrm{FLC}}$ and $\kappa$-FLC index showed similar diagnostic performance in the high category, but not in the low category with inferiority of CSF $\kappa$-FLC and to some extent also of

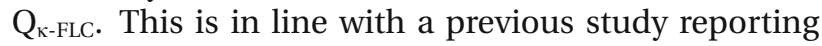
that $\mathrm{Q}_{\mathrm{FLC}}$ depends almost exclusively on the amount of intrathecally synthesized FLC in cases of intrathecal B cell activity (defined by presence of oligoclonal FLC bands), whereas a correlation of $\mathrm{Q}_{\mathrm{alb}}$ and $\mathrm{Q}_{\mathrm{FLC}}$ 


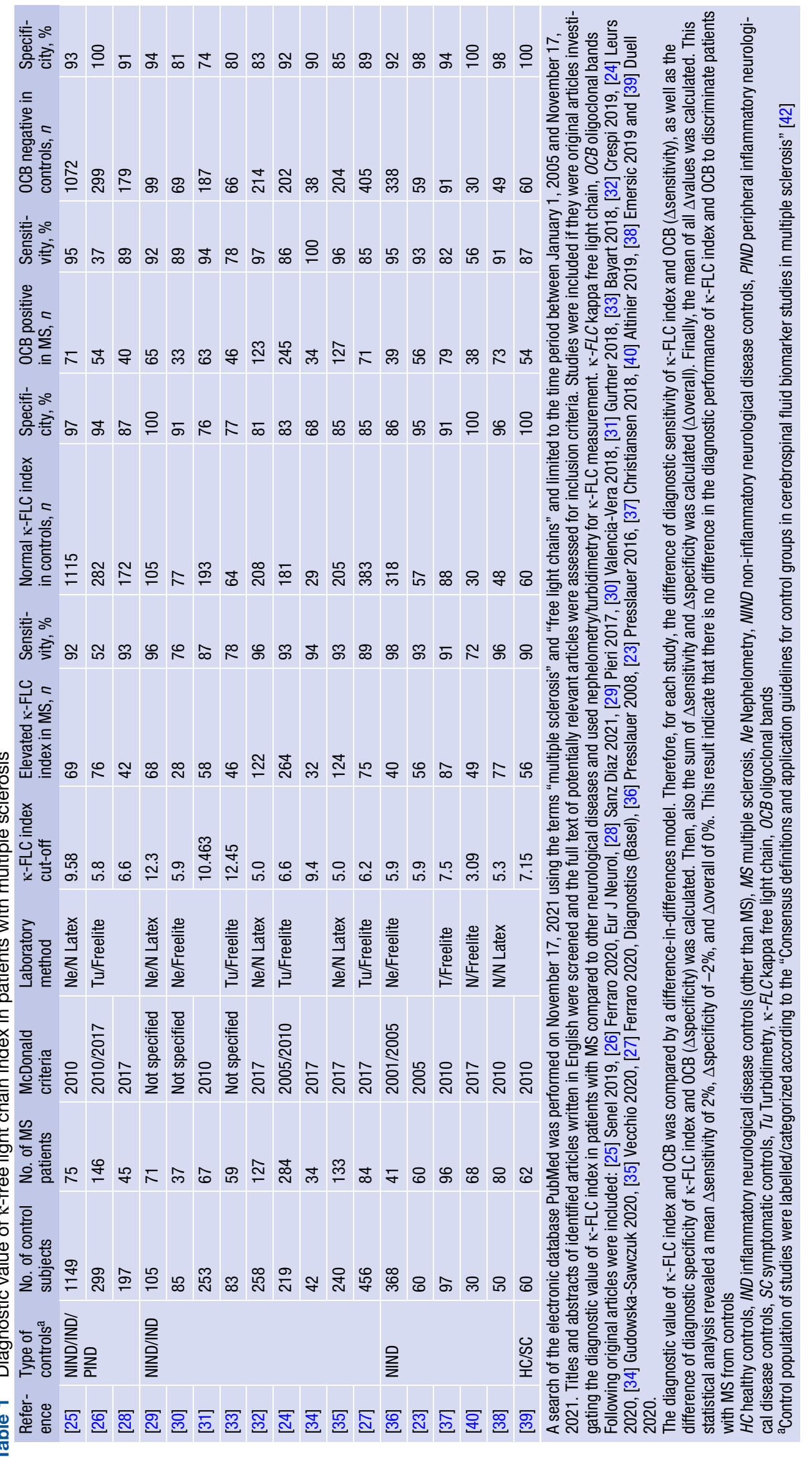




\section{main topic}

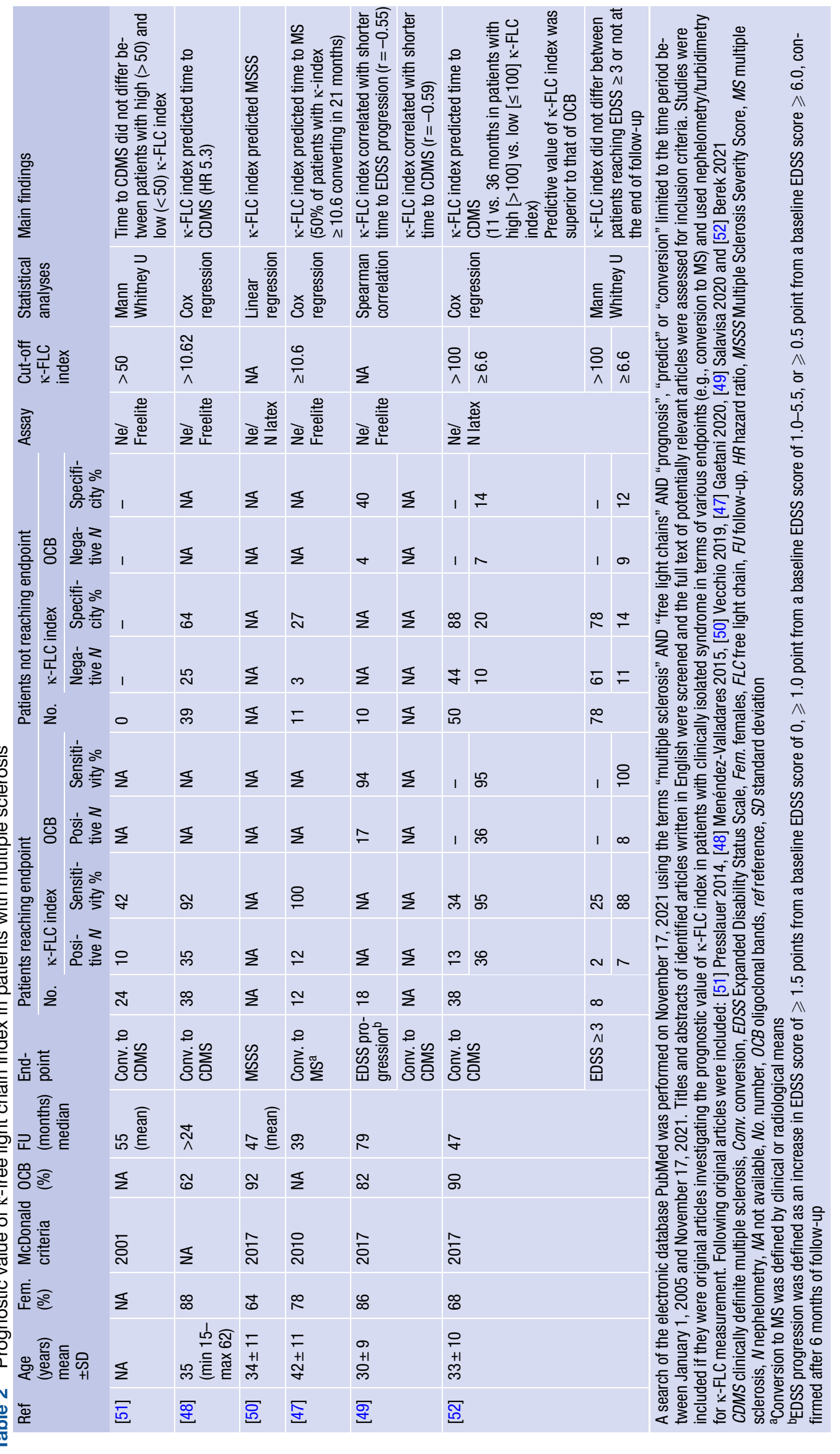


was observed in cases of absent intrathecal B cell activity (defined by negative oligoclonal FLC bands) [56]. Thus, there is evidence that the impact of serum $\kappa$-FLC levels and $\mathrm{Q}_{\mathrm{alb}}$ is negligible in patients with strong intrathecal $\kappa-F L C$ synthesis, but probably not in patients with only low or modest intrathecal $\kappa$-FLC production. Further studies applying multivariate statistics are required to compare these different approaches.

\section{Establishing cut-off values}

Before $\kappa$-FLC index can be introduced into clinical routine, cut-offs have to be established. Different cutoff values might apply depending on the clinical question, e.g., to provide an upper reference limit as determined in a control population (either healthy or e.g. a symptomatic control [42]), to differentiate MS from other IND or to classify patients according to their risk for future disease activity. The so far published cutoff values differentiating MS from other neurological diseases ranged from 3.09 to 12.45 (Table 1). As $\kappa$-FLC index values indeed vary between diseases with high values in MS, followed by other IND and then by nonIND [32, 35, 60], different cut-off values might be useful. For example, one study showed that patients with MS had $\kappa$-FLC index of approximately 90, whereas patients with neuromyelitis optica spectrum disease that is relevant differential diagnosis of MS had values of roughly 20 and control patients values of 4 [60].

Studies that address reproducibility of $\kappa$-FLC index using different assays, platforms and cut-offs between centers are needed, too. Although some work has already been performed in terms of absolute serum $\kappa$-FLC concentrations, this is still lacking for $\kappa$-FLC index. $\kappa$-FLC index might show different robustness, as a ratio (of the CSF/serum $\kappa$-FLC concentration, used for calculation of the $\kappa$-FLC index) is usually less prone to laboratory variations.

\section{Conclusions}

$\kappa$-FLC are a promising biomarker that might replace OCB detection. With regard to its diagnostic value, $\kappa$-FLC index shows a high accuracy similar to that of OCB, but has also significant methodological advantages as an easy, reliable, fast, labor- and cost-saving method. With regard to its prognostic value, the benefit could evolve-either stand alone or in combination with others-to identify early MS patients with a higher risk for further disease activity, e.g., shorter time to a second attack. These patients could be advised to start DMT early or use highly effective DMT, as there is evidence that the time to the second attack has a prognostic impact on long-term disability $[61,62]$ and that early treatment significantly delays conversion to CDMS as well as disability progression [63-65]. Conversely, there is a certain proportion of patients showing a mild disease course who may not need a potentially harmful, psychologically distressing and, last but not least, costly DMT.

Whereas the high diagnostic value is already supported by a multitude of studies, further studies are still required to replicate the independent prognostic value of $\kappa$-FLC index in early MS. Apart from harmonization efforts as depicted above to establish a widely applicable cut-off to definite positivity, potential influential factors such as corticosteroid treatment [52,66], DMT or different disease phases (relapse versus stable remission) on $\kappa$-FLC index also have to be explored.

Thus, there is convincing evidence that $\kappa$-FLC index reliably indicates intrathecal inflammation in MS, might replace OCB determination and probably takes us one step closer to tailored medicine in MS.

Author Contribution H. Hegen participated in drafting the manuscript. K. Berek participated in drafting the manuscript and reviewing the manuscript for intellectual content. F. Deisenhammer participated in reviewing the manuscript for intellectual content.

Funding Open access funding provided by University of Innsbruck and Medical University of Innsbruck.

Conflict of interest $\mathrm{H}$. Hegen has participated in meetings sponsored by, received speaker honoraria or travel funding from Bayer, Biogen, Merck, Novartis, Sanofi-Genzyme, Siemens, Teva, and received honoraria for acting as consultant for Biogen, Novartis and Teva. K. Berek has participated in meetings sponsored by and received travel funding from Roche, Teva and Biogen. F. Deisenhammer has participated in meetings sponsored by or received honoraria for acting as an advisor/speaker for Alexion, Almirall, Biogen, Celgene, Genzyme-Sanofi, Merck, Novartis Pharma, Roche, and Teva. His institution has received research grants from Biogen and Genzyme Sanofi. He is section editor of the MSARD Journal (Multiple Sclerosis and Related Disorders).

Open Access This article is licensed under a Creative Commons Attribution 4.0 International License, which permits use, sharing, adaptation, distribution and reproduction in any medium or format, as long as you give appropriate credit to the original author(s) and the source, provide a link to the Creative Commons licence, and indicate if changes were made. The images or other third party material in this article are included in the article's Creative Commons licence, unless indicated otherwise in a credit line to the material. If material is not included in the article's Creative Commons licence and your intended use is not permitted by statutory regulation or exceeds the permitted use, you will need to obtain permission directly from the copyright holder. To view a copy of this licence, visit http://creativecommons.org/licenses/by/4.0/.

\section{References}

1. Compston A, Coles A. Multiple sclerosis. Lancet. 2002;359(9313):1221-31.

2. Thompson AJ, Banwell BL, Barkhof F, Carroll WM, Coetzee T, Comi G, et al. Diagnosis of multiple sclerosis: 2017 revisions of the McDonald criteria. Lancet Neurol. 2018;17(2):162-73.

3. Arrambide G, Tintore M, Espejo C, Auger C, Castillo M, Río J, et al. The value of oligoclonal bands in the multiple sclerosis diagnostic criteria. Brain. 2018;141(4):1075-84. 
4. Ontaneda D, Tallantyre E, Kalincik T, Planchon SM, Evangelou N. Early highly effective versus escalation treatment approaches in relapsing multiple sclerosis. Lancet Neurol. 2019;18(10):973-80.

5. Hauser SL, Cree BAC. Treatment of multiple sclerosis: a review. Am J Med. 2020; 133(12):1380-1390.e2. https://doi. org/10.1016/j.amjmed.2020.05.049.

6. Giovannoni G. Disease-modifying treatments for early and advanced multiple sclerosis: a new treatment paradigm. Curr Opin Neurol. 2018;31(3):233-43.

7. Weinshenker BG, Bass B, Rice GP, Noseworthy J, Carriere W, Baskerville J, et al. The natural history of multiple sclerosis: a geographically based study. I. Clinical course and disability. Brain. 1989;112(Pt 1):133-46.

8. Bsteh G, Hegen H, Dosser C, Auer M, Berek K, Wurth S, et al. To treat or not to treat: sequential individualized treatment evaluation in relapsing multiple sclerosis. Mult Scler Relat Disord. 2019;(39):101908. https://doi.org/10. 1016/j.msard.2019.101908

9. Tintore M, Rovira A, Río J, Otero-Romero S, Arrambide G, Tur C, et al. Defining high, medium and low impact prognostic factors for developing multiple sclerosis. Brain. 2015;138(Pt7):1863-74.

10. Link H, Tibbling G. Principles of albumin and IgG analyses in neurological disorders. III. Evaluation of IgG synthesis within the central nervous system in multiple sclerosis. Scand J Clin Lab Invest. 1977;37(5):397-401.

11. Reiber H. Flow rate of cerebrospinal fluid (CSF)-a concept common to normal blood-CSF barrier function and to dysfunction in neurological diseases. J Neurol Sci. 1994;122(2):189-203.

12. Auer M, Hegen H, Zeileis A, Deisenhammer F. Quantitation of intrathecal immunoglobulin synthesis-a new empirical formula. Eur J Neurol. 2016;23(4):713-21.

13. Freedman MS, Thompson EJ, Deisenhammer F, Giovannoni G, Grimsley G, Keir G, et al. Recommended standard of cerebrospinal fluid analysis in the diagnosis of multiple sclerosis: a consensus statement. Arch Neurol. 2005;62:865-70.

14. Hegen H,ZinganellA,Auer M, DeisenhammerF.Theclinical significance of single or double bands in cerebrospinal fluid isoelectric focusing. A retrospective study and systematic review. Plos One. 2019;14(4):e215410.

15. Abbas AK, Lichtman AH, Pillai S. Cellular and molecular immunology. 6th ed. 2007: Elsevier Saunders.

16. Nakano T, Matsui $M$, Inoue I, Awata $T$, Katayama S, Murakoshi T. Free immunoglobulin light chain: its biology and implications in diseases. Clin Chim Acta. 2011;412(11/12):843-9.

17. Jones HB. Chemical pathology. Lancet. 1847;2:88-92.

18. Jenner E. Serum free light chains in clinical laboratory diagnostics. Clin Chim Acta. 2014;427:15-20.

19. Bradwell AR, Carr-Smith HD, Mead GP, Tang LX, Showell PJ, Drayson MT, et al. Highly sensitive, automated immunoassay for immunoglobulin free light chains in serum and urine. Clin Chem. 2001;47(4):673-80.

20. TeVelthuisH, KnopI, StamP, van den BroekM, BosHK, HolS, et al. N Latex FLC-new monoclonal high-performance assays for the determination of free light chain kappa and lambda. Clin Chem Lab Med. 2011;49(8):1323-32.

21. Reiber H. Dynamics of brain-derived proteins in cerebrospinal fluid. Clin Chim Acta. 2001;310(2):173-86.

22. Deisenhammer F, Bartos A, Egg R, Gilhus NE, Giovannoni G, Rauer S, et al. Guidelines on routine cerebrospinal fluid analysis. Report from an EFNS task force. Eur J Neurol. 2006;13(9):913-22.
23. Presslauer S, Milosavljevic D, Huebl W, AbouleneinDjamshidian F, Krugluger W, Deisenhammer F, et al. Validation of kappa free light chains as a diagnostic biomarker in multiple sclerosis and clinically isolated syndrome: a multicenter study. Mult Scler. 2016;22(4):502-10.

24. Leurs CE, Twaalfhoven H, Lissenberg-Witte BI, van Pesch V, Dujmovic I, Drulovic J, et al. Kappa freelight chains is a valid tool in the diagnostics of MS: a large multicenter study. Mult Scler. 2020;26(8):912-23.

25. Senel M, Mojib-Yezdani F, Braisch U, Bachhuber F, LewerenzJ, LudolphAC, etal. CSF freelight chains as a marker of intrathecal immunoglobulin synthesis in multiple sclerosis: a blood-CSF barrier related evaluation in a large cohort. Front Immunol Front. 2019;10:641.

26. Ferraro D, Trovati A, Bedin R, Natali P, Franciotta D, Santangelo $\mathrm{M}$, et al. Cerebrospinal fluid kappa and lambda free light chains in oligoclonal band-negative patients with suspected multiple sclerosis. Eur J Neurol. 2020;27(3):461-7.

27. Ferraro D, Bedin R, Natali P, Franciotta D, Smolik K, Santangelo $\mathrm{M}$, et al. Kappa index versus CSF oligoclonal bands in predicting multiple sclerosis and infectious/inflammatory CNS disorders. Diagnostics. 2020;10(10):856.

28. Sanz Diaz CT, de Las Heras Flórez S, Carretero Perez M, Hernández Pérez MÁ, Martín García V. Evaluation of kappa index as a tool in the diagnosis of multiple sclerosis: implementation in routine screening procedure. Front Neurol. 2021;12:676527.

29. Pieri M, Storto M, Pignalosa S, Zenobi R, Buttari F, Bernardini S, et al. KFLC index utility in multiple sclerosis diagnosis: further confirmation. J Neuroimmunol. 2017;309:31-3.

30. Valencia-Vera E, Martinez-Escribano Garcia-Ripoll A, Enguix A, Abalos-Garcia C, Segovia-Cuevas MJ. Application of $\kappa$ free light chains in cerebrospinal fluid as a biomarker in multiple sclerosis diagnosis: development of a diagnosis algorithm. Clin Chem Lab Med. 2018;56(4):609-13.

31. Gurtner KM, Shosha E, Bryant SC, Andreguetto BD, Murray DL, Pittock SJ, et al. CSF free light chain identification of demyelinating disease: comparison with oligoclonal banding and other CSF indexes. Clin Chem Lab Med. 2018;56(7):1071-80.

32. Crespi I, Vecchio D, Serino R, Saliva E, Virgilio E, Sulas MG, et al. Kindex is a reliable marker of Intrathecal synthesis, and an alternative to IgG index in multiple sclerosis diagnostic work-up. JClin Med. 2019;8(4):446.

33. Bayart JL, Muls N, van Pesch V. Free kappa light chains in neuroinflammatory disorders: complement rather than substitute? Acta Neurol Scand. 2018;138(4):352-8.

34. Gudowska-SawczukM, TarasiukJ, KułakowskaA, Kochanowicz J, Mroczko B. Kappa free light chains and IgG combined in a novel algorithm for the detection of multiple sclerosis. Brain Sci Multidiscip Digit Publ Inst. 2020;10(6):324.

35. Vecchio D, Bellomo G, Serino R, Virgilio E, Lamonaca M, Dianzani $U$, etal. Intrathecalkappafreelightchains as markers for multiple sclerosis. Sci Rep. 2020;10(1):20329-20326.

36. Presslauer S, Milosavljevic D, Brücke T, Bayer P, Hübl W, Hübl W. Elevated levels of kappa free light chains in CSF support the diagnosis of multiple sclerosis. J Neurol. 2008;255(10):1508-14.

37. Christiansen M, Gjelstrup MC, Stilund M, Christensen T, Petersen T, Jon Møller H. Cerebrospinal fluid free kappa light chains and kappa index perform equal to oligoclonal bands in the diagnosis of multiple sclerosis. Clin Chem Lab Med. 2018; 57(2):210-20. https://doi.org/10.1515/cclm2018-0400.

38. Emersic A, Anadolli V, Krsnik M, Rot U. Intrathecal immunoglobulin synthesis: the potential value of an adjunct test. Clin Chim Acta. 2019;489:109-16. 
39. DuellF, Evertsson B, Al Nimer F, Sandin Å, OlssonD, OlssonT, et al. Diagnostic accuracy of intrathecal kappa free light chains compared with OCBs in MS. Neurol Neuroimmunol Neuroinflamm. 2020; 57(2):210-220. https://doi.org/10. 1212/NXI.0000000000000775.

40. AltinierS, PuthenparampilM,Zaninotto M, ToffaninE, Ruggero S, Gallo P, et al. Free light chains in cerebrospinal fluid of multiple sclerosis patients negative for IgG oligoclonal bands. Clin Chim Acta. 2019;496:117-20.

41. Dobson R, Ramagopalan S, Davis A, Giovannoni G. Cerebrospinal fluid oligoclonal bands in multiple sclerosis and clinically isolated syndromes: a meta-analysis of prevalence, prognosis and effect of latitude. J Neurol Neurosurg Psychiatry. 2013;84(8):909-14.

42. Teunissen C, Menge T, Altintas A, Álvarez-Cermeño JC, Bertolotto A, Berven FS, et al. Consensus definitions and application guidelines for control groups in cerebrospinal fluid biomarker studies in multiple sclerosis. Mult Scler. 2013;19(13):1802-9.

43. Hegen H, Milosavljevic D, Schnabl C, Manowiecka A, Walde J, Deisenhammer F, et al. Cerebrospinal fluid free light chains as diagnostic biomarker in neuroborreliosis. Clin Chem Lab Med. 2018;56(8):1383-91.

44. Tjernberg I, Johansson M, Henningsson AJ. Diagnostic performance of cerebrospinal fluid free light chains in Lyme neuroborreliosis-a pilot study. Clin Chem Lab Med. 2019;57(12):2008-18.

45. Schwenkenbecher P, Konen FF, Wurster U, Witte T, Gingele S, Sühs K-W, et al. Reiber's diagram for kappa free light chains: the new standard for assessing intrathecal synthesis? Diagnostics. 2019;9(4):194.

46. Schwenkenbecher P, Konen FF, Wurster U, Jendretzky KF, Gingele S, Sühs K-W, et al. The persisting significance of oligoclonal bands in the dawning era of kappa free light chains for the diagnosis of multiple sclerosis. Int J Mol Sci. 2018;19(12):3796.

47. Gaetani L, Di Carlo M, Brachelente G, Valletta F, Eusebi P, Mancini A, et al. Cerebrospinal fluid free light chains compared to oligoclonal bands as biomarkers in multiple sclerosis. J Neuroimmunol. 2020;339:577108.

48. Menéndez-Valladares P, García-Sánchez MI, Cuadri Benítez P, Lucas M, Adorna Martínez M, Carranco Galán V, et al. Freekappalight chains in cerebrospinal fluid as a biomarker to assess risk conversion to multiple sclerosis. Mult Scler J. 2015;1:2055217315620935.

49. Salavisa M, Paixão P, Ladeira AF, Mendes A, Correia AS, Viana JF, et al. Prognostic value of kappa free light chains determinationinfirst-evermultiplesclerosis relapse. JNeuroimmunol. 2020;347:577355.

50. Vecchio D, Crespi I, Virgilio E, Naldi P, Campisi MP, Serino R, et al. Kappa free light chains could predict early disease course in multiple sclerosis. Mult Scler Relat Disord. 2019;30:81-4.

51. Presslauer S, Milosavljevic D, Huebl W, Parigger S, Schneider-Koch G, Bruecke T. Kappa free light chains: diagnostic and prognostic relevance in MS and CIS. Plos One. 2014;9(2):e89945.

52. Berek K, Bsteh G, Auer M, Di Pauli F, Grams A, Milosavljevic D, et al. Kappa free light chains in cerebrospinal fluid predict early multiple sclerosis disease activity. Neurol Neuroimmunol Neuroinflamm. 2021;8(4):e1005.

53. ReiberH,ZemanD, KušnierováP,MundwilerE, BernasconiL Diagnostic relevance of free light chains in cerebrospinal fluid-the hyperbolic reference range for reliable data interpretation in quotient diagrams. Clin Chim Acta. 2019;497:153-62.
54. Makshakov G, Nazarov V, Kochetova O, Surkova E, Lapin S, Evdoshenko E. Diagnostic and prognostic value of the cerebrospinal fluid concentration of immunoglobulin free light chains in clinically isolated syndrome with conversion to multiple sclerosis. Plos One. 2015;10(11):e143375.

55. Senel M, Tumani H, Lauda F, Presslauer S, Mojib-Yezdani R, Otto M, et al. Cerebrospinal fluid immunoglobulin kappa light chain in clinically isolated syndrome and multiple sclerosis. Plos One. 2014;9(4):e88680.

56. Zeman D, Kušnierová P, Bartoš V, Hradílek P, Kurková B, Zapletalová O. Quantitation of free light chains in the cerebrospinal fluid reliably predicts their intrathecal synthesis. Ann Clin Biochem. 2016;53(Pt1):174-6.

57. Duranti F, Pieri M, Centonze D, Buttari F, Bernardini S, Dessi M. Determination of $\kappa F L C$ and $\kappa$ Index in cerebrospinal fluid: a valid alternative to assess intrathecal immunoglobulin synthesis. J Neuroimmunol. 2013;263(1-2):116-20.

58. Vasilj M, Kes VB, Vrkic N, Vukasovic I. Relevance of KFLC quantification to differentiate clinically isolated syndrome from multiple sclerosis at clinical onset. Clin Neurol Neurosurg. 2018;174:220-9.

59. Hegen H, Walde J, Milosavljevic D, Aboulenein-Djamshidian F, Senel M, Tumani H, et al. Free light chains in the cerebrospinal fluid. Comparison of different methods to determine intrathecal synthesis. Clin Chem Lab Med. 2019;57(10):1574-86. https://doi.org/10.1515/cclm-20181300.

60. Cavalla P, Caropreso P, Limoncelli S, Bosa C, Pasanisi MB, Schillaci V, et al. Kappa free light chains index in the differential diagnosis of multiple sclerosis from neuromyelitis optica spectrum disorders and other immune-mediated central nervous system disorders. J Neuroimmunol. 2020;339:577122.

61. Scalfari A, Neuhaus A, Degenhardt A, Rice GP, Muraro PA, Daumer M, et al. The natural history of multiple sclerosis: a geographically based study 10: relapses and long-term disability. Brain. 2010;133(Pt7):1914-29.

62. Tremlett H, Yousefi M, Devonshire V, Rieckmann P, Zhao Y, UBC Neurologists. Impact of multiple sclerosis relapses on progression diminishes with time. Neurology. 2009;73(20):1616-23.

63. Kappos L, Polman CH, Freedman MS, Edan G, Hartung HP, Miller $\mathrm{DH}$, et al. Treatment with interferon beta-1b delays conversion to clinically definite and McDonald MS in patients with clinically isolated syndromes. Neurology. 2006;67(7):1242-9.

64. Comi G, Filippi M, BarkhofF, Durelli L, Edan G, Fernandez O, et al. Effect of early interferon treatment on conversion to definite multiple sclerosis: a randomised study. Lancet. 2001;357(9268):1576-82.

65. Jacobs LD, Beck RW, Simon JH, Kinkel RP, Brownscheidle CM, Murray TJ, et al. Intramuscular interferon betala therapy initiated during a first demyelinating event in multiple sclerosis. CHAMPS Study Group. N Engl J Med. 2000;343(13):898-904.

66. Konen FF, Wurster U, Witte T, Jendretzky KF, Gingele S, Tumani H, et al. The impact of immunomodulatory treatment on kappa free light chains as biomarker in neuroinflammation. Cells. 2020;9(4):842.

Publisher's Note Springer Nature remains neutral with regard to jurisdictional claims in published maps and institutional affiliations. 